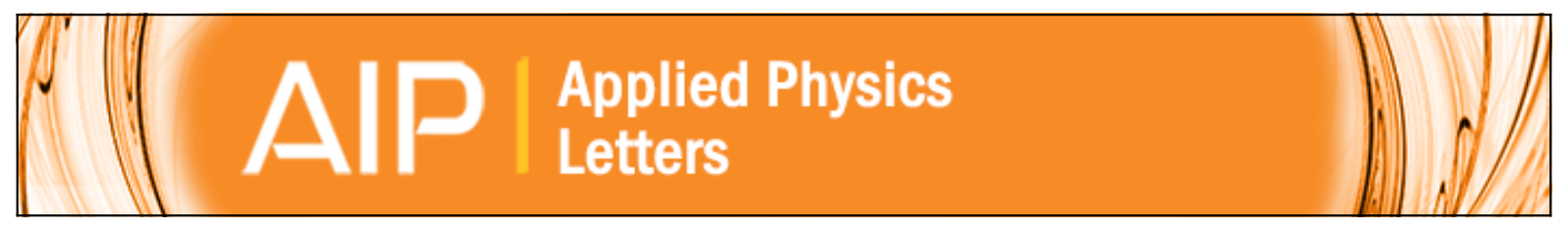

\title{
Relation between growth dynamics and the spatial distribution of intrinsic defects in self-assembled colloidal crystal films
}

G. Lozano and H. Míguez

Citation: Applied Physics Letters 92, 091904 (2008); doi: 10.1063/1.2883943

View online: http://dx.doi.org/10.1063/1.2883943

View Table of Contents: http://scitation.aip.org/content/aip/journal/apl/92/9?ver=pdfcov

Published by the AIP Publishing

\section{Articles you may be interested in}

Optical studies of random disorder of colloidal photonic crystals and its evolution in evaporation induced selfassembly

J. Chem. Phys. 137, 234111 (2012); 10.1063/1.4772095

Selective growth and ordering of self-assembly on metal/polymer thin-film heterostructures via photothermal modulation

Appl. Phys. Lett. 96, 123116 (2010); 10.1063/1.3374322

Spatially controlled reversible colloidal self-assembly

J. Chem. Phys. 131, 134705 (2009); 10.1063/1.3243686

Pressure controlled self-assembly of high quality three-dimensional colloidal photonic crystals Appl. Phys. Lett. 90, 051910 (2007); 10.1063/1.2435613

Modifying the growth of organic thin films by a self-assembled monolayer

J. Appl. Phys. 93, 4852 (2003); 10.1063/1.1559935

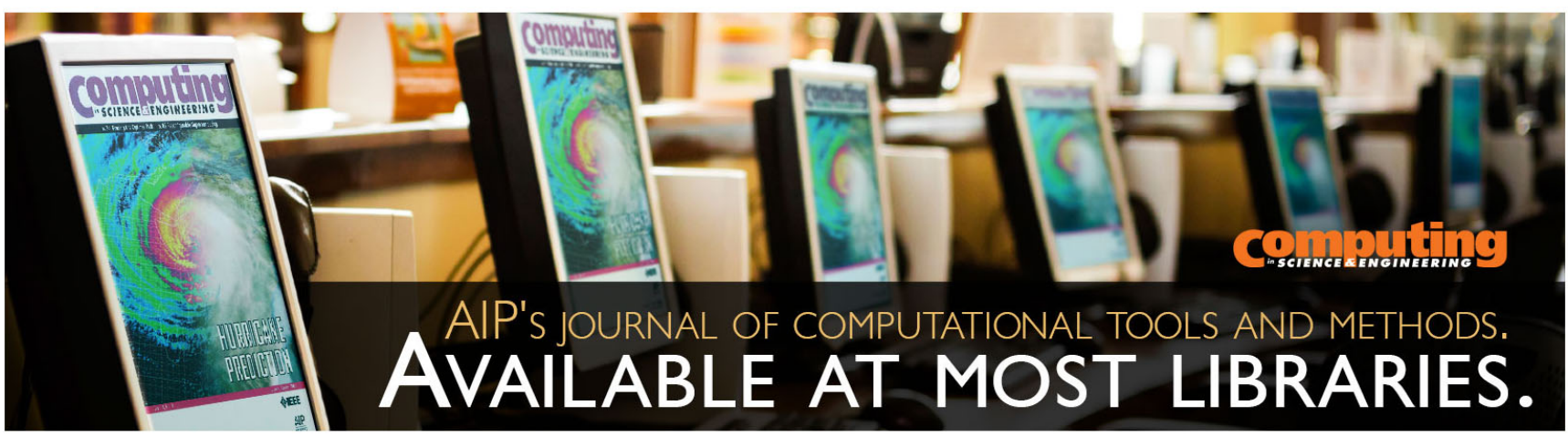




\title{
Relation between growth dynamics and the spatial distribution of intrinsic defects in self-assembled colloidal crystal films
}

\author{
G. Lozano and H. Míguez ${ }^{a}$ \\ Instituto de Ciencia de Materiales de Sevilla, Consejo Superior de Investigaciones Científicas (CSIC), \\ Américo Vespucio 49, 41092 Sevilla, Spain
}

(Received 26 November 2007; accepted 25 January 2008; published online 4 March 2008)

\begin{abstract}
Herein we establish a clear relation between the parameters that govern the growth dynamics and the structural quality of colloidal crystal films. We report an optical analysis of the spatial distribution of intrinsic defects in colloidal crystal films and correlate our results with a theoretical model describing the growth dynamics of such lattices. We find that the amount of defects fluctuates periodically and decreases along the growth direction of the lattice. We demonstrate that these spatial variations are a direct consequence of the temporal oscillations of the crystal film formation velocity, which are inherent to the colloidal particle deposition process. (c) 2008 American Institute of Physics. [DOI: 10.1063/1.2883943]
\end{abstract}

Self-assembled colloidal crystal films ${ }^{1}$ have attracted great attention both as a playground for the growth dynamics and structural analysis of atomlike lattices at the submicron length scale ${ }^{2,3}$ and, in more recent years, as a platform for photonic bandgap research. ${ }^{4}$ For both types of studies, a major drawback is the presence of intrinsic defects that are created, seemingly inescapably, during the crystal formation process. These defects are mainly dislocations, vacancies, stacking faults, and cracks. The presence of such imperfections largely affects the optical properties of these lattices. ${ }^{5,6}$ Although the effect of having different types of intrinsic defects has been studied in some detail both theoretically ${ }^{7,8}$ and experimentally, ${ }^{9-13}$ no strict correlation between the growth dynamics of colloidal crystal films and the presence of imperfections has been established up to date. This is mainly due to the lack of a model describing the colloidal crystal film formation. Recently, we proposed a model that explains the growth dynamics of colloidal crystals by evaporation induced self-assembly onto vertical substrates and found that this dynamics is responsible for the observation of nonuniform thickness profiles, typically oscillatory or steplike, in these films. ${ }^{14}$

Herein we establish for the first time a clear relation between the parameters that govern the growth dynamics and the structural quality of colloidal crystal films. We report an optical analysis of the spatial distribution of intrinsic defects in colloidal crystal films and correlate our results with a theoretical model describing the growth dynamics of such lattices. We find that the amount of defects fluctuates periodically and decreases along the growth direction of the lattice. We demonstrate that these spatial variations are a direct consequence of temporal oscillations of the crystal film formation velocity, which are inherent to the colloidal particle deposition process. The period and the amplitude of these correlated damped oscillations are determined by the experimental conditions under which the lattices are deposited.

Self-assembled three dimensional colloidal crystal films were deposited onto vertical, flat glass substrates by evaporation of $700 \mathrm{~nm}$ diameter polystyrene sphere suspensions

${ }^{a}$ Author to whom correspondence should be addressed. Electronic mail: hernan@icmse.csic.es.
(IKERLAT, average diameter of $700 \mathrm{~nm}$, polydispersity below $3 \%$, density $\rho=1.1 \mathrm{~g} / \mathrm{cm}^{3}$, refractive index $n=1.59$ ) with particle volume fraction between $0.1 \%$ and $0.2 \%$. Evaporation temperature was kept constant for a given lattice growth process and ranged between 35 and $50{ }^{\circ} \mathrm{C}$. As the suspension evaporates, a crystalline film is deposited on the substrate at the contact line with the suspension meniscus. ${ }^{15,16}$ These films display photonic crystal properties, which we make use of in extracting detailed information on their structure. ${ }^{14}$ Optical reflectance microspectroscopy measurements were performed using a Fourier transform infrared spectrophotometer (BRUKER IFS-66) attached to a microscope. A $10 \times$ objective with a numerical aperture of 0.1 (light cone angle of $\pm 5.7^{\circ}$ ) was used to irradiate the lattices and collect the reflected light at quasinormal incidence with respect to its surface, the [111] of a face centered cubic (fcc) lattice. ${ }^{1}$ A spatial filter was used to selectively detect light from $100 \times 100 \mu \mathrm{m}^{2}$ square regions of the sample. In Fig. 1(a), we show a typical series of reflectance spectra measured from these spots at regularly spaced intervals of $100 \mu \mathrm{m}$ along the growth direction. In each spectrum, a clear maximum is observed at frequencies corresponding to the photonic pseudogap that opens up at the $L$ point of the fcc
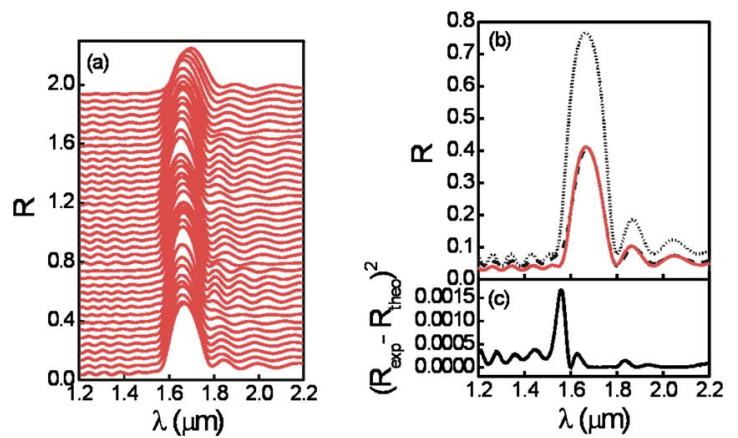

FIG. 1. (Color online) (a) Normal incidence specular reflectance spectra taken every $0.1 \mathrm{~mm}$ along the growth direction, from $0.1 \mathrm{~mm}$ side square spots. These curves have been vertically shifted for clarity. (b) Measured (red solid line) and calculated specular reflectance spectra (dashed and dotted lines). The dashed line is the optimum fitting attained for $\operatorname{Im}(n)=0.014$, and the dotted line is the reflectance spectrum that would result if $\operatorname{Im}(n)$ $=0$. (c) Squared difference between experimental and theoretical reflectance spectra for the optimum fitting. 
lattice. A number of side lobes of much lower intensity can also be detected. These are Fabry-Pérot oscillations whose number and spectral separation are determined, in a first approximation, by the average refractive index and finite thickness of the film.

Reflectance results were fitted using a code based on the scalar wave approximation. ${ }^{17}$ Central to the analysis reported herein is the fact that real films present imperfections that cause light to be scattered diffusely, removing part of the energy from the specularly reflected or forward-transmitted beams. This extinction is modeled here by adding an imaginary part in the refractive index of the spheres $[\operatorname{Im}(n)]$ of our crystal, which accounts for all possible sources of losses in the lattice. Thus, the model structure employed is a fcc lattice of spheres of refractive index $n=1.59+\operatorname{Im}(n)$ in a medium of refractive index $n=1$. In the calculations, we consider the number of close packed monolayers (i.e., with film thickness $H$ ) and the imaginary part of the refractive index of the spheres $[\operatorname{Im}(n)]$ as the only variables. An optimum fitting was attained for each spectrum using the method of squared minima. In Fig. 1(b), we plot an example of one of these fitting spectra along with the corresponding experimental spectrum (black dashed and red solid lines, respectively). Figure 1(c) shows the squared difference between them, which is minimized in order to select the optimum fitting. It should be remarked that the introduction of extinction in the calculation is absolutely necessary to accurately reproduce the experimental peak shape. By no means does a close packed lattice of purely real refractive index spheres yield the measured intensity and width of the experimental reflectance maximum. To illustrate the magnitude of the effect $\operatorname{Im}(n)$ has on the calculated optical properties, in Fig. 1(b), we plot the reflectance calculated using the same fitting parameters and constants of for the optimum fitting (black dashed line) but considering $\operatorname{Im}(n)=0$ instead of $\operatorname{Im}(n)$ $=0.014$. The resulting curve (black dotted line) presents a similar number of secondary lobes and shape but a much larger maximum peak intensity.

Both the crystal thickness $(H)$ and imaginary part of the refractive index of the spheres $[\operatorname{Im}(n)]$ were extracted at each measured spot, allowing us to attain detailed profiles of both independent variables along the growth direction. In Fig. 2 we plot the experimental thickness profiles (scatter graphs) versus time for three different colloidal crystal films. Oscillatory or steplike film thickness profiles are always observed on top of a linearly increasing background, a very similar behavior being detected along different parallel lines along the growth direction. The fluctuations observed in these profiles are the consequence of the motion of the contact line between the meniscus and the substrate, which results, in turn, from the inherent variations of the friction force acting on it, as has been demonstrated in a model describing the growth dynamics of such films reported in Ref. 14. The fittings of the experimental profiles shown in Fig. 2 (black solid lines) were obtained by using an equation derived within this model,

$$
H(t)=C_{1} t+\frac{C_{2}}{v_{g}(t)},
$$

where $C_{1}$ and $C_{2}$ are constants and $v_{g}(t)$ is the crystal growth velocity, defined as the time derivative of the position of the contact line,

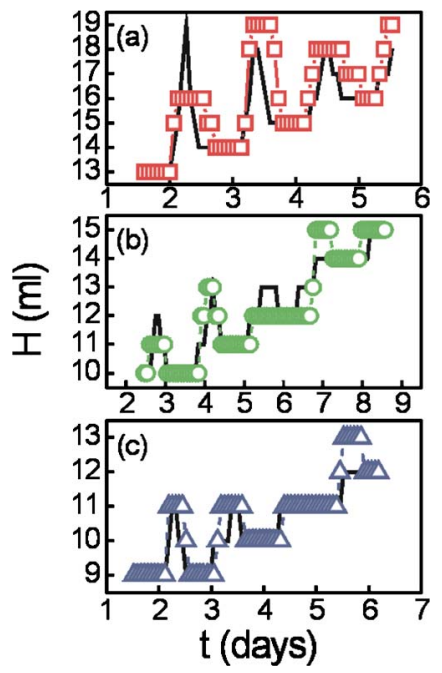

FIG. 2. (Color online) Experimental (colored symbols) and theoretical (black solid line) thickness profiles of colloidal crystals vertically deposited at $35{ }^{\circ} \mathrm{C}$ from $700 \mathrm{~nm}$ diameter polystyrene latex suspensions of (a) $0.2 \%$, (b) $0.15 \%$, and (c) $0.1 \%$ particle volume fractions.

$$
R(t)=R_{0}+V_{S}\left[t-\frac{1}{\mu} e^{-\lambda t} \sin (\mu t)\right]
$$

where $R_{0}$ is the position of the contact line at $t=0, V_{S}$ is the speed of the contact line attained when the steady state is reached $(t \rightarrow \infty)$, and $\mu$ and $\lambda$ are the period and the extinction parameter of the periodic fluctuations of $R(t)$, respectively. The dependence of $V_{S}, \mu$, and $\lambda$ on physical variables can be found in Ref. 14. After Eq. (2) and taking into account the values of the fitting parameters extracted from the simulated thickness profiles, $v_{g}(t)$ can be approximated by

$$
v_{g}(t) \simeq V_{S}\left[1-e^{-\lambda t} \cos (\mu t)\right] .
$$

This model has been proven to account for all film thickness profiles observed in crystals grown under very different conditions, which supports its validity and generality.

Since no optical absorption occurs in the photon energy range under analysis, the extinction factor $[\operatorname{Im}(n)]$ must account exclusively for the optical losses in the form of diffuse scattering caused by imperfections in the lattice. We can then establish a relation between its magnitude and the density of intrinsic defects in the film. It should be kept in mind that using this approach, we will not find a quantitative understanding of the nature of such defects since we approximate the joint effect of all the different types of the defects in $\operatorname{Im}(n)$. In Fig. 3, $\operatorname{Im}(n)$ profiles are plotted (scatter graphs) for the three different films whose thickness profiles were plotted and fitted in Fig. 2. Interestingly, in spite of being a totally independent variable, $\operatorname{Im}(n)$ also shows, systematically, fluctuations with a well-defined period. A closer look reveals that the amplitude of the said fluctuations decreases with time (i.e., along the growth direction). The corresponding time variation of the crystal growth speed attained from the fittings and given by Eq. (3) is also drawn. The comparison explicitly shows that there is a strong correlation between the magnitude of $\operatorname{Im}(n)$ required to fit the spectra and the crystalline film growth speed. Interestingly, those parts of the film that are formed at the slowest speed present the lowest number of imperfections, while those that are deposited faster are more defective. Also, the damping of the os- 


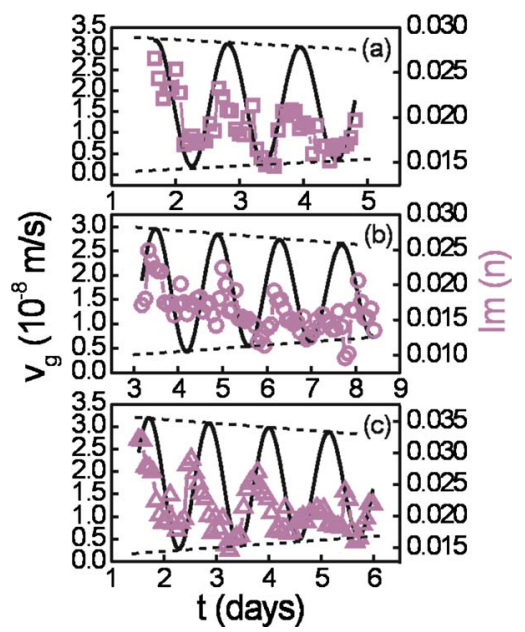

FIG. 3. (Color online) Time evolution of both crystal growth velocity $v_{g}$ (black solid line) and the experimentally estimated imaginary part of the refractive index $\operatorname{Im}(n)$ (scatter graphs) extracted from the fitting of the optical reflectance of colloidal crystals vertically deposited at $35^{\circ} \mathrm{C}$ from $700 \mathrm{~nm}$ polystyrene latex suspensions of (a) $0.2 \%$, (b) $0.15 \%$, and (c) $0.1 \%$ particle volume fractions. The dashed lines are guides only for the eye to show the damping of the oscillations of $v_{g}$.

cillations seen for $\operatorname{Im}(n)$ is also observed for the amplitude of the velocity fluctuations, further confirming their correlation.

Insight on the relation between the parameters that govern the growth dynamics and the structural quality of colloidal crystal films can be extracted from Fig. 4. In this figure, we plot the contact line position $R(t)$ [see Fig. 4(a)], film thickness $H(t)$ [see Fig. 4(b)], film growth velocity $v_{g}(t)$ [see Fig. 4(c)], and the extinction factor $\operatorname{Im}(n)$ [see Fig. 4(c)] versus time for a whole thickness profile oscillation. It can be seen that while the contact line position remains nearly constant with time, the crystal growth velocity is gradually reduced until it reaches a minimum. As a consequence, the thickness of the forming film increases until it reaches a maximum. It is interesting to notice that the areas that present less degree of disorder are those that grew at the slower speed, i.e., those formed when the array's growing edge advanced at a lower rate. Besides, it can be observed that the larger the acceleration of the contact line, the more defective is the formed film, as it happens in the regions between minimum and maximum thickness plateaus. Thus, the stick-slip motion of the contact line strongly affects the structure of the forming film and its optical quality. It should be noted that the amount of disorder present in colloidal crystal films has previously been approximated by assuming an optical extinction factor that accounts for imperfections in the lattice. ${ }^{18,19}$ The most remarkable result of these two precedents was that the amount of disorder is smaller for thicker sample. Our study indicates that the thicker regions of a particular film are indeed expected to be less defective since they grow more slowly. In this regard, our analysis allows us to propose a physical origin for previously reported observations. The decisive factor that affects crystal quality is the crystal growth velocity, which also determines the crystal thickness.

In conclusion, we have established for the first time a clear relation between the parameters that govern the growth dynamics and the structural quality of colloidal crystal films. We have demonstrated that the amount of intrinsic defects present in self-assembled colloidal crystal films fluctuates

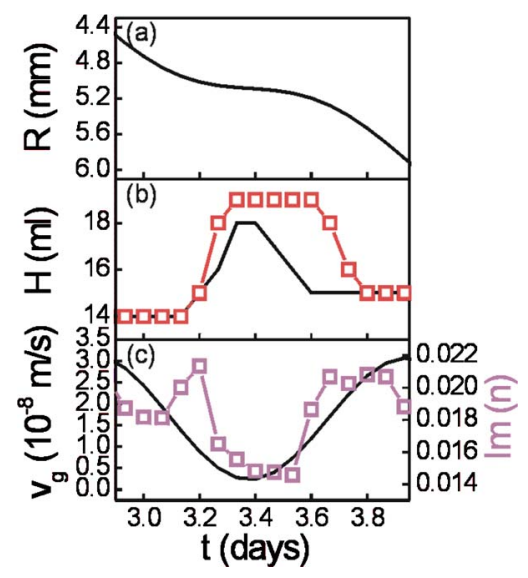

FIG. 4. (Color online) Time evolution of the (a) contact line position, (b) experimental (red squares) and calculated (black solid line) thickness profiles, (c) and calculated crystal growth velocity (black solid line) and the experimentally estimated imaginary part of the refractive index $\operatorname{Im}(n)$ (violet squares). The colored lines linking the experimental data are just a guide for the eye.

periodically along the growth direction as a result of the temporal periodic fluctuation of the lattice growth velocity, a phenomenon that is inherent to the evaporation induced selfassembly process. Those parts of the film that were formed at the slowest speed are thicker and present the lowest number of imperfections. Our work opens the door to conscious design of experiments to improve self-assembled colloidal crystal film quality through the control of its deposition speed.

This work has been funded by the Spanish Ministry of Science and Education under Grant No. MAT2004-03028. G.L. thanks CSIC for funding through a Ph.D. scholarship.

${ }^{1}$ J. F. Bertone, P. Jiang, K. S. Hwang, D. M. Mittleman, and V. L. Colvin, Phys. Rev. Lett. 83, 300 (1999).

${ }^{2}$ F. Ramiro-Manzano, F. Meseguer, E. Bonet, and I. Rodríguez, Phys. Rev. Lett. 97, 028304 (2006).

${ }^{3}$ P. Pieranski, L. Strzelecki, and B. Pansu, Phys. Rev. Lett. 50, 900 (1983).

${ }^{4}$ N. Tétreault, H. Míguez, and G. A. Ozin, Adv. Mater. (Weinheim, Ger.) 16, 1471 (2004).

${ }^{5}$ E. Palacios-Lidón, B. H. Juárez, E. Castillo-Martinez, and C. López, J. Appl. Phys. 97, 063502 (2005).

${ }^{6}$ L. A. Dorado, R. A. Depine, and H. Míguez, Phys. Rev. B 75, 241101 (2007).

${ }^{7}$ V. Yannopapas, N. Stefanou, and A. Modinos, Phys. Rev. Lett. 86, 4811 (2001).

${ }^{8}$ A. F. Koenderink, A. Lagendijk, and W. L. Vos, Phys. Rev. B 72, 153102 (2005).

${ }^{9}$ B. Gates and Y. Xia, Appl. Phys. Lett. 78, 3178 (2001).

${ }^{10}$ X. Checoury, S. Enoch, C. López, and A. Blanco, Appl. Phys. Lett. 90, 161131 (2007).

${ }^{11}$ R. Rengarajan, D. M. Mittleman, C. Rich, and V. L. Colvin, Phys. Rev. E 71, 016615 (2005).

${ }^{12}$ Y. A. Vlasov, V. N. Astratov, A. V. Baryshev, A. A. Kaplyanskii, O. Z. Karimov, and M. F. Limonov, Phys. Rev. E 61, 5784 (2000).

${ }^{13}$ J. F. Galisteo-López, E. Palacios-Lidón, E. Castillo-Martinez, and C. López, Phys. Rev. B 68, 115109 (2003).

${ }^{14}$ G. Lozano and H. Míguez, Langmuir 23, 9933 (2007).

${ }^{15}$ A. S. Dimitrov and K. Nagayama, Langmuir 12, 1303 (1996).

${ }^{16}$ P. Jiang, J. F. Bertone, K. S. Hwang, and V. L. Colvin, Chem. Mater. 11, 2132 (1999)

${ }^{17}$ D. M. Mittleman, J. F. Bertone, P. Jiang, K. S. Hwang, and V. L. Colvin, J. Chem. Phys. 111, 345 (1999).

${ }^{18}$ J. F. Galisteo-López, M. Galli, M. Patrini, A. Balestreri, L. C. Andreani, and C. López, Phys. Rev. B 73, 125103 (2006).

${ }^{19}$ L. A. Dorado, R. A. Depine, G. Lozano, and H. Míguez, Phys. Rev. B 76, 245103 (2007). 